\title{
Efecto analgésico y sobre la neuroconducta de la interacción entre tramadol y diclofenaco en dosis escalonada en ratones
}

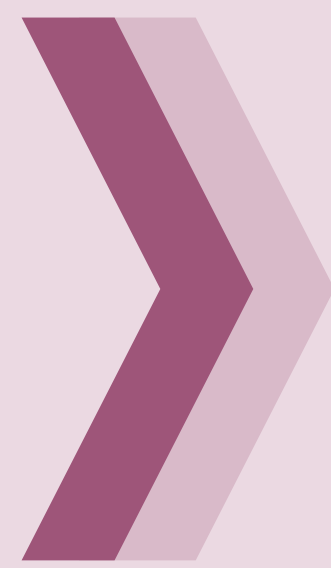

\author{
Analgesic and neurobehavioral \\ effect of the interaction between \\ Tramadol and Diclofenac in \\ escalated doses in mice
}

\author{
Alberto Alcibiades Salazar-Granara, 1,2,3,4,a \\ Lorena Torres-Acosta ${ }^{1,2,4, b}$ Andrea Siles de la Portilla, ${ }^{1,2,4, b}$ \\ Stephanie Palacios-Ramírez, ${ }^{1,2,4, b}$ \\ Carlos Alberto Vergara-Ascenzo, ${ }^{1,2,4, b}$ \\ Carlos Torres-Angulo, ${ }^{1,2,4,}$ Carlos Pante-Medina ${ }^{1,2,3,4,}$
}
1. Facultad de Medicina Humana de la Universidad San Martín de Porres (FMH-USMP).
2. Centro de Investigación de Medicina Tradicional y Farmacología de la FMH-USMP.
3. Bioterio de la FMH-USMP.
4. Laboratorio docente de Farmacología de la FMH-USMP.
a. Médico. Doctor en Medicina.
b. Estudiante de Medicina Humana.
c. Técnico especialista en investigación biomédica.

\section{RESUMEN}

Objetivo. Determinar el efecto sobre el dolor y el sistema nervioso de la interacción entre tramadol y diclofenaco, en dosis escalonadas. Materiales y métodos. Se utilizaron 60 ratones albinos, distribuidos en diez grupos; cuatro grupos fueron de interacción y recibieron $V O$ tramadol $10 \mathrm{mg} / \mathrm{Kg}$ y diclofenaco en dosis escalonada $(10,15,20$ y $25 \mathrm{mg} / \mathrm{Kg})$. Seis grupos recibieron VO tramadol, $10 \mathrm{mg} / \mathrm{Kg}$; diclofenaco, $10 \mathrm{mg} / \mathrm{Kg}$; diazepam, $25 \mathrm{mg} / \mathrm{Kg}$; cafeina, $25 \mathrm{mg} / \mathrm{Kg}$ y agua destilada, 0,1 mL/10 g; este último grupo no recibió medicamentos. Las sustancias se administraron una hora antes de la inducción del dolor. Se emplearon la prueba de contorsiones abdominales, para evaluar el dolor, y la prueba de Irwin, para el sistema nervioso. Se evaluaron el porcentaje de inhibición de contorsiones abdominales, porcentaje de prolongación del periodo de latencia, número de contorsiones abdominales y período de latencia. Se aplicaron las pruebas ANOVA de una cola, Tukey y correlación de Pearson. Para la prueba de Irwin se aplicó la prueba de Fisher. Resultados. Los porcentajes de inhibición de las contorsiones abdominales fueron de 55,1\%; 89,9\%; 74,5\% y $94,5 \%$ en los grupos de interacción $2,4,7$ y 10, respectivamente, frente a $31,6 \%$ (tramadol), $19,4 \%$ (diclofenaco) y $4,8 \%$ (control). El periodo de latencia fue de 609,$7 ; 891,2 ; 860,5$ y 1100 segundos en los grupos de interacción $2,4,7$ y 10, respectivamente, frente a 479,7 (tramadol), 281,8 (diclofenaco) y 475,7 segundos (control). La prueba de ANOVA demostró diferencias significativas ( $p<0,05$; IC 95\%) con relación al porcentaje de inhibición de contorsiones y el periodo de latencia. La prueba de Irwin evidenció piloerección, sedación, aumento de la respiración, incoordinación motora y cola de Straub. Conclusiones. Se comprobó el efecto analgésico sinérgico de la coadministración de tramadol en dosis fija y diclofenaco en dosis escalonada.

Palabras clave: analgésicos; tramadol; diclofenaco; sinergismo farmacológico

\begin{abstract}
Objective. Determine the effect on pain and nervous system of the interaction between tramadol and diclofenac, in escalating doses. Materials and methods. 60 albino mice, divided into ten groups were used; four groups were for interaction and they received tramadol VO $10 \mathrm{mg} / \mathrm{Kg}$ and diclofenac in staggered doses $(10,15,20$ and $25 \mathrm{mg} / \mathrm{Kg})$. Six groups received tramadol $10 \mathrm{mg} / \mathrm{Kg}$; diclofenac $10 \mathrm{mg} / \mathrm{Kg}$; diazepam, $25 \mathrm{mg} /$ $\mathrm{Kg}$; caffeine, $25 \mathrm{mg} / \mathrm{Kg}$ and distilled water, $0.1 \mathrm{~mL} / 10 \mathrm{~g}$; this last group received no medication. The substances were administered one hour before pain induction. Writhing test assessed the effect on pain and Irwin test evaluated the nervous system. The statistical validation of the writhing inhibition percentage, latency percentage, number of writhes and latency was performed using ANOVA one tail, Tukey and Pearson correlation. For Irwin test, Fisher's test was applied. Results. The percentage of writhing inhibition were $55.1 \%$, $89.9 \%, 74.5 \%$ and $94.5 \%$ by interaction groups $2,4,7$ and 10 , respectively, versus $31.6 \%$ (tramadol), 19.4\% (diclofenac) and $4.8 \%$ (control). The latency period was 609.7; 891.2; 860.5 and 1100 seconds by interaction groups 2, 4, 7 and 10, respectively, versus 479.7 (tramadol), 281.8 (diclofenac) and 475.7 seconds (control). ANOVA showed significant differences ( $p<0.05$; $\mathrm{Cl} 95 \%$ ) relative to the percent inhibition of contortions and the latency period. Irwin test showed piloerection, sedation, increased respiration, motor incoordination and Straub tail. Conclusions. Synergistic analgesic effect of co-administration of fixed dose tramadol and diclofenac dose escalation was found.
\end{abstract}

Key words: analgesics; tramadol; diclofenac; drug synergism 


\section{INTRODUCCIÓN}

El uso racional de medicamentos corresponde a la administración adecuada de la medicación según las necesidades clínicas de los pacientes, es decir, durante un periodo apropiado, en dosis adecuadas y al menor costo posible. ${ }^{1}$ Sin embargo, según cifras de la Organización Mundial de la Salud (OMS), más del $50 \%$ de medicamentos se prescriben, dispensan o venden de manera inapropiada y no son consumidos correctamente por la mitad de los pacientes. ${ }^{1}$ En el Perú existe la libre obtención de fármacos sin prescripción médica, que podría estar generando un problema de salud pública, pues son utilizados ignorando los efectos secundarios. Lo antes mencionado se ratifica en un estudio que demuestra que entre el 40 y $60 \%$ de la población se automedica y que el $55 \%$ del dinero de los hogares es invertido en la compra de medicinas directamente en farmacias y/o boticas. ${ }^{2}$

Otra investigación demuestra que el grupo más importante son los antiinflamatorios, con ventas que ascienden al $7 \%$ del total del mercado nacional. ${ }^{3}$ Por otra parte, en pacientes que fueron operadas por cesárea, se ha comprobado que la combinación de diclofenaco y tramadol por vía intramuscular fue más efectiva que la monoterapia, para la hiperalgesia primaria y secundaria. ${ }^{4}$ Asimismo, la combinación de diclofenaco y tramadol fue efectiva para la analgesia posoperatoria en pacientes de cirugía maxilofacial, inclusive se observó una disminución del uso de analgésicos posoperatorios. ${ }^{5}$

En animales de experimentación, la interacción de naproxeno y tramadol, mediante un análisis isobolográfico, demostró un efecto analgésico y de sinergismo, al reducir las contorsiones abdominales en el roedor; ; asimismo, la interacción del diclofenaco y el tramadol, en el modelo de la inmersión de la cola, reveló una antinocicepción y potenciación significativa. También, es reconocida la actividad del tramadol en la neuroconducta, al incrementar los niveles de serotonina, dopamina y noradrenalina en el sistema nervioso; ${ }^{8}$ además, existen estudios que comprueban los efectos de las prostaglandinas como neuroprotectores frente a convulsiones y reguladores del ciclo de sueñovigilia, entre otros. ${ }^{9-13}$
La mayoría de las investigaciones de la interacción de analgésicos opioides y no opioides, carece de la delimitación de la relación dosis-efecto, y de la potencial concomitancia de los efectos colaterales en el sistema nervioso central. Existen modelos experimentales en roedores que permiten explorar el efecto de sustancias químicas, como la prueba de contorsiones abdominales, ${ }^{14}$ que sirve para determinar el efecto analgésico, y la prueba de Irwin, que permite explorar la neuroconducta. ${ }^{14}$

Este estudio se centró en explorar el efecto de la interacción entre tramadol a dosis fija y diclofenaco en dosis escalonada sobre el dolor y la neuroconducta en el roedor.

\section{MATERIALES Y MÉTODOS}

Estudio experimental, preclínico, prospectivo y triple ciego; realizado en el Centro de Investigación de Medicina Tradicional y Farmacología de la Facultad de Medicina Humana de la Universidad de San Martín de Porres (FMH-USMP); durante el periodo febrero a noviembre de 2014. La muestra la conformaron 60 ratones albinos hembra (Mus musculus) adquiridos en el bioterio del Instituto Nacional de Salud (INS); los ratones pasaron un proceso de aclimatación en las instalaciones del bioterio de la FMH-USMP, en condiciones estándares de niveles de temperatura de 22 ${ }^{\circ} \mathrm{C}\left(+/-2{ }^{\circ} \mathrm{C}\right)$, humedad relativa promedio entre 45 a $70 \%$ y niveles de ruido menores de $70 \mathrm{~dB}$. Fueron aislados en jaulas con libre acceso a agua y se les proporcionó alimento balanceado. La distribución y asignación aleatorizada de los grupos experimentales se hizo por sorteo. ${ }^{15} \mathrm{La}$ muestra química estuvo compuesta por diazepam, ampollas de $10 \mathrm{mg} / 2 \mathrm{~mL}$; diclofenaco, ampolla de $75 \mathrm{mg} / 3 \mathrm{~mL}$; tramadol, ampolla de $50 \mathrm{mg} / 1 \mathrm{~mL}$; agua destilada y cafeína, $100 \mathrm{mg}$.

Para la evaluación primaria neurofarmacológica se empleó la prueba de Irwin, durante primera hora de la prueba, en intervalos de 15 minutos, a los 15, 30, 45 y 60 minutos. Esta consistió en la observación de manifestaciones neurológicas en el ratón después de la administración de una sustancia. Se evaluó la presencia o ausencia de letalidad, convulsiones, cola de Straub,* sedación, excitación, marcha anormal (en círculos o en puntas de pie), saltos, 
incoordinación motora, piloerección, estereotipias (oler, masticar o movimientos de cabeza), contorsiones abdominales, sacudidas de cabeza, escozor y alteración de la respiración. ${ }^{16}$

Para la prueba de contorsiones abdominales en roedores se utilizó el modelo de dolor visceral por ácido acético (prueba nociceptiva). Cada animal recibió una inyección intraperitoneal (ip) de 0,1 $\mathrm{mL} / 10 \mathrm{~g}$ de peso. Los animales fueron colocados por separado en jaulas, para luego observar y contar el número de contorsiones abdominales (distensión exagerada del abdomen y estiramiento de las patas traseras). Se registró el número total de contorsiones durante los 20 minutos siguientes a la inyección de ácido acético. Los grupos experimentales recibieron las sustancias de estudio por vía oral, una hora antes de la inducción de dolor. ${ }^{17}$

Se conformaron diez grupos experimentales, con seis ratones cada uno: grupo 1, diclofenaco $10 \mathrm{mg} /$ $\mathrm{Kg}$; grupo 3, tramadol; grupo 5, placebo (agua destilada) 0,1 mL/10 g de peso corporal; grupo 6, diazepam, $32 \mathrm{mg} / \mathrm{Kg}$; grupo 8, control, no se le administró ninguna sustancia; grupo 9, cafeína, $32 \mathrm{mg} /$ Kg. A los grupos 10, 7, 2 y 4, se les administró una dosis estable de $10 \mathrm{mg}$ de tramadol más una dosis escalonada de diclofenaco de 10, 15, 20 y $25 \mathrm{mg} /$ $\mathrm{Kg}$ respectivamente. La administración de las sustancias fue por vía oral.

Se aplicó un sistema de triple ciego para la administración de las sustancias químicas, observación de manifestaciones y análisis estadístico:15 asimismo, se realizó un entrenamiento virtual previo para la evaluación de las manifestaciones de dolor de los ratones, empleando los programas Virtual Pharmacology Lab ${ }^{\odot} 18$ y Microlabs $^{\odot}, 19$ y también un ensayo práctico con un roedor vivo. Por otra parte, se controlaron la humedad, la temperatura y el ruido de la sala de experimentación empleando un higrómetro digital Thermo-Hygro@, marca VWR International ( $\mathrm{T}^{\circ}: 5-34^{\circ} \mathrm{C}$; humedad: $32-82 \%$ ), un sensor de ruido, marca Digital Sound Level Me$\operatorname{ter}^{\odot}(60-120 \mathrm{~dB})$, y dos termoventiladores portátiles modelo NF15C, marca IMACO ${ }^{\circ}$.
El estudio contó con la aprobación del Instituto de Investigación de la FMH-USMP, que se basó en los principios y los lineamientos para las investigaciones en animales de laboratorio.20, 21

Los datos se presentan como frecuencias, media y desviación estándar, según corresponde. Para los datos cuantitativos se aplicó la prueba de ANOVA de una cola (luego de haber cumplido los requisitos para su uso) y la prueba de pareo de Tukey. Para los datos cualitativos se aplica la prueba exacta de Fisher. Se estableció un nivel de significancia estadística de $p<0,05$, con un intervalo de confianza de $95 \%$. Se usaron los programas informáticos Microsoft Excel Windows $2010^{\circ}$ y GraphPad Prism ${ }^{\oplus}$ versión 5.

\section{RESULTADOS}

Al analizar el porcentaje de inhibición de las contorsiones mediante la prueba de ANOVA de una cola, se obtuvo un valor $p<0,05$. Por otra parte, la correlación de Pearson muestra una relación inversamente proporcional entre dosis y efecto respecto al efecto analgésico $(r=-0,09 ; p=0,6502$; IC 95\%: -0,48 a 0,32; coeficiente de determinación $r^{2}=0,009519$ ) (Tabla 1).

Asimismo, para el número de contorsiones, la prueba de ANOVA de una cola determinó un valor $p<0,05$. Por otro lado, la prueba de correlación de Pearson muestra una relación directamente proporcional entre dosis y efecto, respecto al efecto nociceptivo ( $r=0,97 ; p=0,6502$; IC 95\%: $-0,32$ a 0,48 ; coeficiente de determinación $r^{2}=$ 0,009519) (Figura 1).

Para el período de latencia en segundos, se realizó la prueba de ANOVA, que muestra un valor $p<$ 0,05 entre períodos de latencia por fármaco y dosis administrada ( $p=0,0014)$. La correlación de Pearson mostró una relación inversamente proporcional de dosis y efecto, respecto al efecto nociceptivo ( $r=-0,3 ; p=0,1946$; IC: $95 \%$ : $-0,6$ a 0,15 ; coeficiente de determinación $r^{2}=0,07523$ ) (Tabla 1). 


\section{Auta Méäica Pernana}

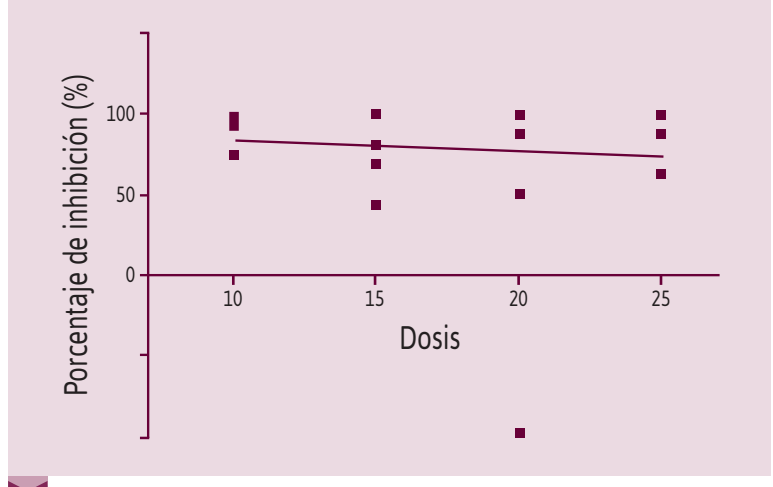

Figura 1. Efecto de la interacción de tramadol $10 \mathrm{mg} / \mathrm{Kg}$ y diclofenaco en dosis escalonadas $(10,15,20$ y $25 \mathrm{mg} / \mathrm{Kg})$ sobre la inhibición de las contorsiones abdominales.

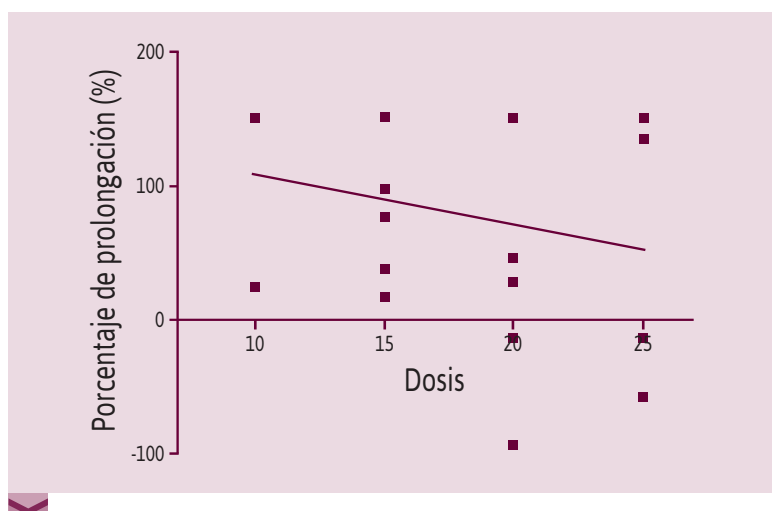

Figura 2. Efecto de la interacción de tramadol $10 \mathrm{mg} / \mathrm{Kg}$ y diclofenaco en dosis escalonadas $(10,15,20$ y $25 \mathrm{mg} / \mathrm{Kg})$ sobre el porcentaje de prolongación del periodo de latencia de las contorsiones abdominales.

\begin{tabular}{|c|c|c|c|c|c|c|c|c|c|c|}
\hline \multirow{2}{*}{ Grupo } & \multirow{2}{*}{$\begin{array}{l}\text { Sustancia/ } \\
\text { fármaco }\end{array}$} & \multirow{2}{*}{$\mathrm{N}$} & \multicolumn{2}{|c|}{$\begin{array}{l}\text { Número de } \\
\text { contorsiones }\end{array}$} & \multicolumn{2}{|c|}{$\begin{array}{l}\text { Porcentaje de inhibición de } \\
\text { contorsiones abdominales }\end{array}$} & \multicolumn{2}{|c|}{$\begin{array}{l}\text { Periodo de latencia } \\
\text { en segundos }\end{array}$} & \multicolumn{2}{|c|}{$\begin{array}{l}\text { Porcentaje de prolongación } \\
\text { del periodo de latencia }\end{array}$} \\
\hline & & & Media & $\sigma$ & Media & $\sigma$ & Media & $\sigma$ & Media & $\sigma$ \\
\hline 8 & Control & 6 & 16,3 & 8,5 & 4,8 & 52,2 & 475,7 & 278,6 & $-7,9$ & 58,6 \\
\hline 5 & $\begin{array}{l}\text { Agua destilada } \\
(1 \mathrm{mg} / 10 \mathrm{ml})\end{array}$ & 6 & 12,3 & 5,7 & 24,5 & 34,8 & 444,0 & 402,3 & $-6,6$ & 84,6 \\
\hline 1 & Diclofenaco (10 mg) & 6 & 13,2 & 11,3 & 19,4 & 69,0 & 281,8 & 172,8 & $-40,8$ & 36,3 \\
\hline 3 & Tramadol (10 mg) & 6 & 11,7 & 3,5 & 31,6 & 21,4 & 479,7 & 156,3 & 0,8 & 32,9 \\
\hline 10 & $\begin{array}{l}\text { Tramadol }(10 \mathrm{mg})+ \\
\text { diclofenaco }(10 \mathrm{mg})\end{array}$ & 6 & 0,8 & 1,6 & 94,5 & 9,8 & 1100,0 & 244,9 & 131,3 & 51,5 \\
\hline 2 & $\begin{array}{l}\text { Tramadol (10 mg) + } \\
\text { diclofenaco (20 mg) }\end{array}$ & 6 & 7,3 & 12,4 & 55,1 & 76,1 & 609,7 & 384,9 & 87,4 & 95,8 \\
\hline 7 & $\begin{array}{l}\text { Tramadol }(10 \mathrm{mg})+ \\
\text { diclofenaco }(15 \mathrm{mg})\end{array}$ & 6 & 4,2 & 3 & 74,5 & 18,3 & 860,5 & 229,4 & 80,9 & 48,2 \\
\hline 4 & $\begin{array}{l}\text { Tramadol (10 mg) + } \\
\text { diclofenaco (25 mg) }\end{array}$ & 6 & 1,7 & 2,3 & 89,9 & 14,3 & 891,2 & 455,8 & 28,2 & 80,9 \\
\hline
\end{tabular}

La prueba de ANOVA reveló un valor $p<0,05(p<0,0001)$ para el porcentaje de inhibición de las contorsiones abdominales entre los grupos 1, 3, $10,2,7$ y 4 . 
Con respecto al porcentaje de prolongación del periodo de latencia, la correlación de Pearson muestra una relación inversamente proporcional entre dosis y efecto, respecto al efecto analgésico $(r=-0,3 ; p=0,1946$; IC 95\%: -0,6 a 0,15; coeficiente de determinación $r^{2}=0,07523$ ) (Figura 2).

Con la prueba de Tukey se obtuvo un valor $p<0,05$ en los resultados obtenidos entre el grupo 1 frente a los grupos 6 y 10 y el grupo 9 contra los grupos 3 y 10, en relación con el periodo de latencia y el porcentaje de prolongación del periodo de latencia.

Por otra parte, la prueba de pareo de Tukey arrojó un valor $p>0,05$ entre los grupos 2, 4, 7 y 10 frente a los grupos 1 y 3 , en relación con el porcentaje de inhibición de las contorsiones abdominales y el porcentaje de prolongación del periodo de latencia.

En la figura 3 se presentan las frecuencias de sedación en la prueba de Irwin, cuantificadas en intervalos de tiempo. No se observó una diferencia significativa de los grupos 10, 2, 4, y 7 frente al grupo de diazepam $(p>0,05)$.

Por otra parte, en la figura 4 se puede observar la excitación en los distintos grupos. Se pudo ver que al comparar el grupo 9 (cafeína $32 \mathrm{mg} / \mathrm{Kg}$ ) con los grupos de dosis escalonadas $(10,7,2$ y 4$)$ se obtuvo un valor $p<0,0001$.

Además, en la figura 5 se observó que los grupos 10,2 , y 4 frente al grupo 3 (tramadol), mostraron un valor $p>0,05$ relacionado con la presencia de cola de Straub. Asimismo, al comparar el grupo 7 con el grupo 3 se obtuvo un valor de $p=0,0479$.

También, en la figura 6 se observó que el grupo 10 en comparación con el grupo 8 (control) mostró un valor $p>0,05$ con respecto a la respiración, en donde el grupo control mostró un aumento de la frecuencia respiratoria. En contraste, el grupo 7 frente al grupo 8 obtuvo un valor $p=0,0012$, en donde se observa una disminución de la frecuencia respiratoria. Además, en el grupo 2 se obtuvo un valor $p=0,0128$, representado por un aumento de la frecuencia respiratoria. En el grupo 4 se obtuvo un valor $p=0,0017$ y no se observaron cambios.

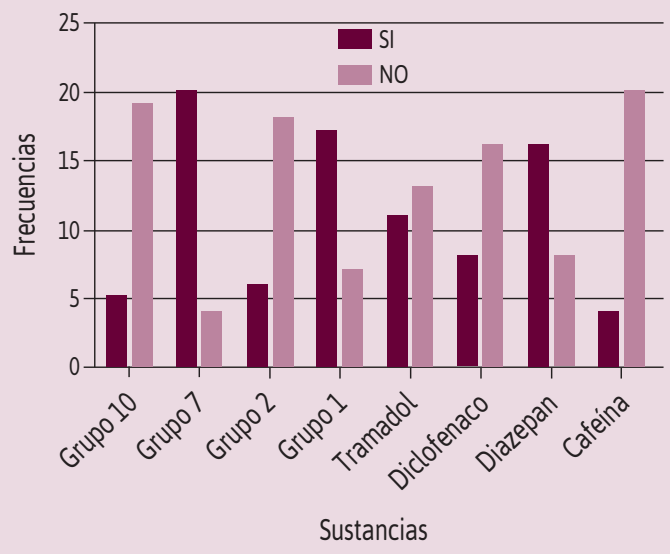

Figura 3. Frecuencias absolutas del efecto sedativo de las diversas sustancias empleadas.

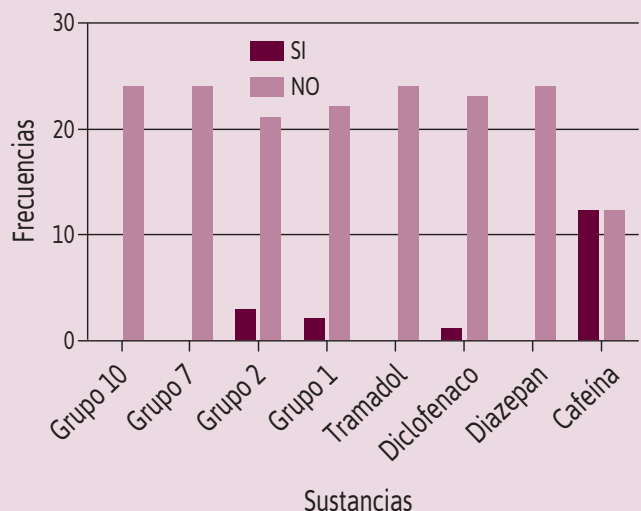

Figura 4. Frecuencias absolutas del efecto excitatorio de las sustancias

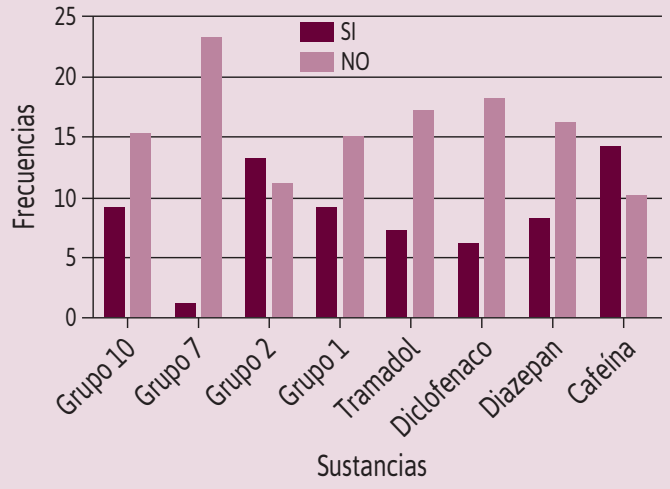

Figura 5. Frecuencias absolutas de la presencia de cola de Straub ocasionada por las sustancias. 


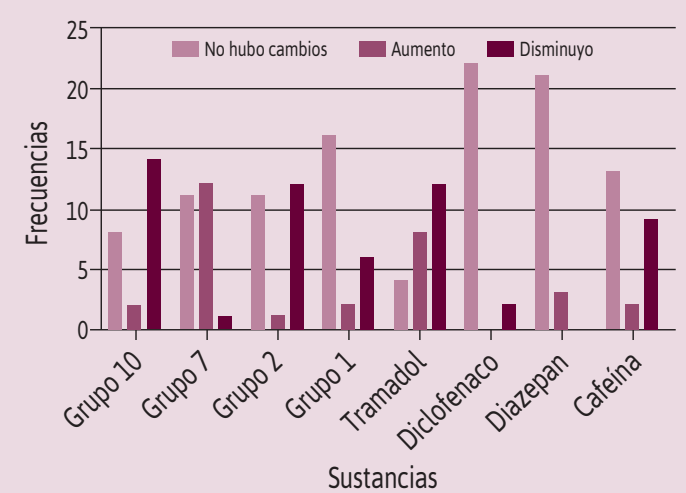

Figura 6. Frecuencias absolutas del efecto de las sustancias sobre la respiración.

\section{DISCUSIÓN}

En este estudio se observó que la interacción entre tramadol en dosis establecida y diclofenaco en dosis escalonada produjeron un efecto analgésico superior a los efectos individuales; esto permite plantear un posible efecto de sinergismo, ${ }^{7}$ que, por sus mecanismos de acción, correspondería al de potenciación. 22,23

Las bases moleculares que explicarían el efecto de sinergismo observado en este estudio son que el tramadol actúa en la vía opioide típica, bloqueando la recaptación de noradrenalina, que ejercería su actividad agonista sobre los receptores alfa 2 adrenérgicos, produciendo una retroalimentación negativa. ${ }^{24}$ Asimismo, el diclofenaco activa receptores alfa 2 a nivel espinal y supraespinal y tiene interacción significativa con receptores opioides a nivel espinal. Por otra parte, el diclofenaco, al inhibir la síntesis de prostaglandinas, disminuye la sensibilización de los nociceptores periféricos. ${ }^{25}$

La relación dosis-efecto presentó una tendencia de curva negativa; sin embargo, no fue significativa, lo cual se explica por la distribución de los datos, que se presentó en varios grupos como no gaussiana (Tabla 1). Este tipo de distribución puede deberse a interacciones farmacocinéticas, como el polimorfismo genético, o diferencias a nivel del CYP3A4, enzima que participa en el metabolismo de ambos fármacos. ${ }^{26}$
Asimismo, por medio de la prueba de Irwin se observó la presencia concomitante de sedación en los grupos interacción. Dado que en las guías clínicas para el manejo del dolor se recomienda sedar al paciente para calmar su dolencia, ${ }^{27}$ esto podría ser una ventaja para cumplir con las recomendaciones del manejo del dolor.

El efecto de la sedación, relacionado con la actividad del diclofenaco, es contradictorio debido a que existen estudios que indican un efecto sedante por la acción de prostaglandinas E2 y $\mathrm{D} 2{ }^{28}$ lo cual propicia la hipótesis de la acción del AINE en otros niveles como el histaminérgico o el gabaérgico, planteando un desequilibrio en la homeostasis de los diversos autacoides. ${ }^{29}$

Por otro lado, el efecto analgésico de la coadministración de los fármacos en este estudio se puede sustentar por la presencia de cola de Straub, debido a que existen estudios que comprobaron que los opioides generan este efecto concomitante. ${ }^{30}$ Asimismo, se observó piloerección en la interacción, que indica actividad del sistema neurovegetativo, que se sustenta por el agonismo de los receptores alfa 2 adrenérgicos. ${ }^{25}$ También se presentó alteración de la respiración, que probablemente es un mecanismo compensatorio ante la inhibición de prostaglandinas bronco-dilatadoras como la PGE2. ${ }^{31}$

Una de las principales limitaciones de este estudio fue que no se pudo delimitar el nivel y mecanismo de acción, eventos que recomendamos explorar en futuros estudios.

\section{CONCLUSIONES}

Se demostró efecto de sinergismo en la actividad analgésica entre tramadol y diclofenaco en dosis escalonadas. Asimismo, se observó la presencia de efectos neuroconductuales concomitantes: sedación, cola de Straub, piloerección y respiración anormal.

\section{AGRADECIMIENTOS}

Al doctor Frank Lizarazo Cáparo, decano de la Facultad de Medicina Humana de la Universidad de San Martín de Porres, por su gran apoyo y colaboración, y al doctor Benjamín Castañeda Castañeda, director del Instituto de Investigación de la FMH-USMP. 


\section{REFERENCIAS BIBLIOGRÁFICAS}

I. Organización Mundial de la Salud. Promoción del uso racional de medicamentos: componentes centrales [Internet]. Ginebra, Suiza: OMS; 2002 [citado: 8 nov 2014]. Disponible en: http://apps. who.int/medicinedocs/pdf/s4874s/s4874s.pdf.

2. República del Perú, Ministerio de Salud, Dirección General de Medicamentos, Insumos y Drogas (DIGEMID). Manual para la enseñanza de uso racional de medicamentos a la comunidad [Internet]. Lima, Perú: Ministerio de Salud; 2005 [citado 24 mar 2014]. Disponible en: http://www.digemid.minsa.gob.pe/ UpLoad\%5CUpLoaded\%5CPDF/l al 12.pdf

3. Miranda JJ. El mercado de medicamentos en el Perú: ¿libre o regulado? [Internet]. Lima, Perú: Consorcio de Investigación Económica y Social (CIES); 2004 [citado I 4 nov 20I4]. Disponible en: http://www.mef.gob.pe/contenidos/pol_econ/documentos/ Medicamentos_competencia.pdf

4. Wilder-Smith CH, Hill L, Dyer RA, Torr G, Coetzee E. Postoperative sensitization and pain after cesarean delivery and the effects of single im doses of tramadol and diclofenac alone and in combination. Anesth Analg. 2003;97(2):526-33.

5. Roux S, Sablé E, Porsolt RD. Primary observation (Irwin) test in rodents for assessing acute toxicity of a test agent and its effects on behavior and physiological function. Curr Protoc Pharmacol. 2005; Chapter 10: Unit 10.10. p. 2616-38.

6. Satyanarayana PS, Jain NK, Singh A, Kulkarni SK. Isobolographic analysis of interaction between cyclooxygenase inhibitors and tramadol in acetic acid-induced writhing in mice. Prog Neuropsychopharmacol Biol Psychiatry. 2004;28(4):64I-9.

7. Melo-RuizV,De Jesús-ContrerasA, Oviedo-BecerrilA, RodríguezRamírez R, Rosas-Trejo E, García-Núñez M. Evaluación de los efectos antinociceptivos en la coadministración de tramadol con diclofenaco vía intraperitoneal en ratones. Enf Neurol. 201I; I0(I):II-5.

8. Bertram G. Katzung, Susan B. Masters, Anthony J. Trevor. Farmacología básica y clínica. I I. ${ }^{a}$ ed. Barcelona, España: McGraw Hill; 2010.

9. Kaushik MK, Aritake K, Kamauchi S, Hayaishi O, Huang ZL, Lazarus $M$, et al. Prostaglandin $D(2)$ is crucial for seizure suppression and postictal sleep. Exp Neurol. 20I4;253:82-90.

10. Urade Y, Hayaishi O. Prostaglandin D2 and sleep/wake regulation. Sleep Med Rev. 20I I;I5(6):4I I-8.

II. Salvadori MG, Banderó CR, Jesse AC, Gomes AT, Rambo LM, Bueno LM, et al. Prostaglandin E(2) potentiates methylmalonateinduced seizures. Epilepsia. 2012;53(I):189-98.

12. Jiang J, Quan Y, Ganesh T, Pouliot WA, Dudek FE, Dingledine R. Inhibition of the prostaglandin receptor EP2 following status epilepticus reduces delayed mortality and brain inflammation. Proc Natl Acad Sci U S A. 20 I3; I I0(9):359 I-6.

13. Jiang J, Ganesh T, Du Y, Quan Y, Serrano G, Qui M, et al. Small molecule antagonist reveals seizure-induced mediation of neuronal injury by prostaglandin E2 receptor subtype EP2. Proc Natl Acad Sci U S A. 20I2;109(8):3|49-54.

14. Vogel G. Drug Discovery and Evaluation: Pharmacological Assays. Frankfurt, Germany: Springer; 2006.

15. Hernandez R, Fernandez C, Baptista P. Metodología de la investigación. 5. ${ }^{a}$ ed. México: McGraw-Hill; 2010.
16. Pugsley MK, Authier S, Curtis MJ. Principles of safety pharmacology. Br J Pharmacol. 2008; I54(7): 1382-99.

17. Vogel HG. Drug Discovery and Evaluation: Pharmacological Assays. 2. ${ }^{\text {a }}$ ed. Berlin, Germany: Springer-Verlag; 2002.

18. Mujica J, Pulliti Y. Procesamiento de señales in vivo, edición audiovisual y creación de un prototipo de software educativo para simulación de experimentos en farmacología [Tesis para optar el grado de Bachiller en Ingeniería Electrónica]. Lima, Perú: Universidad San Martín de Porres; 2009.

19. van Wilgenburg H. Microlabs for Pharmacologists [Computer based courses in Pharmacology]. Amsterdam, The Netherlands: University of Amsterdam; 2014 [citado 4 nov 20I4]. Disponible en: http://oslovet.norecopa.no/produkt.aspx?.produkt=538I.

20. Asociación Médica Mundial. Declaración de la AMM sobre el uso de animales en la investigación biomédica (adoptada por la 4I. a Asamblea Médica Mundial, Hong Kong, setiembre 1989 y revisada por la 57. Asamblea General de la AMM, Pilanesberg, Sudáfrica, octubre 2006) [Internet]. Ferney-Voltaire, Francia: AMM [fecha de acceso: 9 jul 20l4]. Disponible en: http://www. wma.net/es/30publications/I0policies/al8/

21. Council for International Organizations of Medical Sciences. International guiding principles for biomedical research involving animals [Internet]. Diciembre 2012. Geneva, Switzerland: CIOMS; 2012 [citado 30 junio 20I4] Disponible en: http://grants.nih.gov/ grants/olaw/Guiding_Principles_20I2.pdf

22. Hardman JG, Limbird LE, Gilman AG. Goodman \& Gilman: Las bases farmacológicas de la terapéutica. 12. ${ }^{a}$ ed. New York: McGraw-Hill; 20II. p. 508-I0.

23. Hardman JG, Limbird LE, Gilman AG. Goodman \& Gilman: Las bases farmacológicas de la terapéutica. 12.a ed. New York: McGraw-Hill; 20II. P. 986-7.

24. Stone LS, MacMillan LB, Kitto KF, Limbird LE, Wilcox GL. The alpha2a adrenergic receptor subtype mediates spinal analgesia evoked by alpha 2 agonists and is necessary for spinal adrenergicopioid synergy. J Neurosci. 1997; I7(I8):7157-65.

25. Mobasher MA, Sajedianfard J, Jamshidzadeh A, Naghdi N, Namvaran MM. The effects of tramadol on norepinephrine and MHPG releasing in locus coeruleus in formalin test in rats: a brain stereotaxic study. Iran J Basic Med Sci. 20|4;17(6):4 |9-25.

26. Wang D, Sadee W. The making of a CYP3A biomarker panel for guiding drug therapy.J Pers Med. 2012;2(4): I75-91.

27. Neira F., Ortega J. L. Guías de práctica clínica en el tratamiento del dolor: una herramienta en la práctica clínica. Rev Soc Esp Dolor. 2008; I5(6):399-4I3.

28. Gilmore DP, Shaikh AA. The effect of prostaglandin E2 in inducing sedation in the rat. Prostaglandins. 1972;2(2): I43-5I.

29. Gomes JC, Pearce FL. Comparative studies on the effect of non-steroidal anti-inflammatory drugs (NSAID) on histamine release from mast cells of the rat and guinea pig.Agents Actions. | 988:24(3-4):266-7|.

30. King T, Gardell LR, Wang R, Vardanyan A, Ossipov MH, Malan $\mathrm{TP} \mathrm{J}$, et al. Role of NK-I neurotransmission in opioid-induced hyperalgesia. Pain. 2005; I 16(3):276-88.

31. Mathé AA, Hedqvist P. Effect of prostaglandins F2 alpha and E2 on airway conductance in healthy subjects and asthmatic patients.Am Rev Respir Dis. 1975; I I (3):3 3-20.

\section{Correspondencia}

Dra. Lorena Torres-Acosta

Itorresacosta@gmail.com

Fecha de recepción: 6 de noviembre de 2014

Fecha de aceptación: 28 de mayo de 2015

\section{Conflictos de interés}

Los autores declaran no tener conflictos de interés durante el planteamiento, ejecución de la investigación y la elaboración del artículo para su publicación 